\title{
Capillary Electrophoresis-Electrospray Mass Spectra of the Herbicides Paraquat and Diquat
}

\author{
Xinbei Song \\ Oak Ridge Institute for Science and Education, Cincinnati, Ohio \\ William L. Budde \\ U.S. Environmental Protection Agency, Office of Research and Development, National Exposure Research \\ Laboratory, Cincinnati, Ohio
}

\begin{abstract}
The positive ion electrospray mass spectra of the quaternary ammonium salt herbicides paraquat and diquat are examined by on-line separation with capillary electrophoresis (CE) and by direct infusion of the analytes. The analytes are separated by $C E$ in 7-10 $\mathrm{min}$ at $\mathrm{pH}$ 3.9 in $50 \%$ methanol-water by using several different separation buffer electrolytes. The capillary electrophoresis-electrospray ionization (CE-ES) mass spectra of paraquat and diquat consist primarily of doubly charged molecular ions, singly charged molecular ions, and singly charged deprotonated ions. The direct infusion spectra consist primarily of doubly charged molecular ions and singly charged deprotonated ions. The relative abundances of the doubly charged and deprotonated ions depend strongly on the presence or absence of ammonium ion in the CE separation buffer or the direct infusion solution. A deprotonation mechanism is proposed in which the free base ammonia is the deprotonating agent in the desolvating charged droplets or in the gas phase. The analytical potential of the CE-ES electrospray approach for environmental analyses is evaluated in terms of the precision of replicate injections, linear concentration range, and estimated detection limit. ( $J$ Am Soc Mass Spectrom 1996, 7, 981-986)
\end{abstract}

$\mathrm{P}$ araquat and diquat are herbicides used to produce desiccation and defoliation on contact. Paraquat (1,1'-dimethyl-4,4'-bipyridinium dichloride) and diquat [6,7-dihydrodipyrido(1,2a:2' $1^{\prime}$-c)pyrazinedium dibromidel have been used in large quantities in the United States to control terrestrial and aquatic weeds. The compounds have similar structures; each has two quaternary nitrogens. In the United States the maximum contaminant level for diquat in drinking water is $20 \mu \mathrm{g} / \mathrm{L}$. Paraquat currently is not regulated in drinking water, but its use is restricted in the United States. According to a recent report [1] paraquat may be the highest volume banned or restricted-use pesticide exported by the United States (7228 tons in 1994).

Address reprint requests to Dr. William L. Budde, USEPA, 26 West Martin Luther King Drive, Cincinnati, $\mathrm{OH} 45268$.
Paraquat dication, $m / z=93$ :<smiles>C[n+]1ccc(-c2cc[n+](C)cc2)cc1</smiles>

Diquat dication, $m / z=92$ :<smiles></smiles>

Because both compounds have commercial and environmental importance, their mass spectra are of considerable interest as a basis for identification in environmental media. The NIST/EPA/NIH Mass Spectra Database [2] of nominal 70-eV electron ionization (EI) spectra (PC version 4 for Microsoft DOS) does not contain a diquat spectrum, but does contain three entries that correspond to paraquat and several closely 
related substances. The entries are labeled paraquat dichloride (CASRN 1910-42-5), 1,1'-dimethyl-4,4'-bipyridinium (CASRN 4685-14-7), and 4,4'-bis-methylpyridinium diiodide (no CASRN, but database 37115 ). The cations are the same in the three substances and the anions are disclosed in two of them.

All three paraquat EI spectra show evidence of thermal decomposition and all are of very limited value for environmental analyses. It is reasonable to assume the paraquat spectra were measured by heating the ionic solid samples in a direct insertion probe because the doubly charged quaternary ammonium cation would be expected to be relatively nonvolatile (the sample introduction technique is not given in the database). Several of these spectra do contain the possibility ( $\sim 2 \%$ relative abundance) of a diagnostically important doubly charged cation, but these weak signals are just as likely the result of chemical or electronic noise. Environmental analyses invariably require some kind of on-line separation of the analytes from the sample matrix and other specific substances in the sample, but this capability is not available with a direct insertion probe. For the determination of paraquat and diquat in environmental media a new approach is needed that incorporates a high resolution separation and high sensitivity, and provides definitive mass spectra to support reliable identifications.

During a search for a definitive technique to identify and measure paraquat and diquat in environmental samples, we examined their positive ion electrospray (ES) mass spectra. Because these two herbicides are present as ion pairs or partly dissociated ions in solution, capillary electrophoresis (CE) appeared to be the perfect separation technique, which allowed the study of their electrospray spectra under identical conditions. Smith et al. [3] reported high efficiency separations for a group of simple, singly charged alkyl quaternary ammonium salts by using capillary electrophoresis-electrospray/mass spectrometry (CEES/MS). We observed singly and doubly charged molecular ions and deprotonated singly charged ions $\left[\mathrm{M}^{2+}-\mathrm{H}^{+}\right]^{+}$in the otherwise simple spectra of both paraquat and diquat. However, the relative abundances of the doubly charged and deprotonated ions depended on the nature of the electrolyte in the CE separation buffer or the direct infusion solution. Smith et al. [3] previously noted a dependence of mass spectra on the buffer composition in CE-ES/MS.

\section{Experimental}

\section{Materials}

Paraquat and diquat were obtained from the U.S. Environmental Protection Agency's pesticide repository with 98-99\% purity and were used without further purification. Methanol and acetic acid were Fisher Scientific (Fair Lawn, NJ) high-performance liquid chromatography grade, and phenol and all buffer electrolytes (Fisher Scientific) were used without purification.

\section{Equipment}

Capillary electrophoresis was conducted by using an ATI Unicam Crystal 300 series instrument at ambient temperature. For the measurements of migration times only, a Linear Instruments Corp. (Reno, NV) model UVIS 200 detector at $210 \mathrm{~nm}$ was employed. The electrospray interface was an Analytica of Branford (Branford, CT) model 101546-4, serial number 1002, equipped with the CE-PRO ${ }^{\mathrm{TM}}$ probe. This interface has a design similar to the design of Fenn et al. [4] in which the fused silica capillary electrospray tip is held at ground potential and the high voltage (HV) is applied to surrounding cylindrical electrodes and the Pt-coated ends of a glass capillary inlet to the mass spectrometer. A Harvard Apparatus (South Natick, MA) model 55-1111 syringe pump was used to supply a constant flow of sheath liquid to the capillary electrophoresis-electrospray (CE-ES) probe. The mass spectrometer was a Hewlett-Packard (Palo Alto, CA) model 5988 quadrupole instrument connected to a Technivent (St. Louis, MO) data system.

\section{Capillary Electrophoresis-Electrospray / Mass Spectrometry}

The CE was conducted by using a $90-\mathrm{cm} \times 375-\mu \mathrm{m}$ o.d. $\times 75-\mu \mathrm{m}$-i.d. uncoated fused silica capillary (Polymicro Technologies, Phoenix, AZ). All the CE separation buffers consisted of about $0.5-1.5 \%$ acetic acid in $50 \%(\mathrm{v} / \mathrm{v})$ methanol-water. All the buffer solutions, except as noted in subsequent text, also contained another electrolyte at $5 \mathrm{mM}$ (Tables 1 and 2). The buffer designated acetic acid in Tables 1 and 2 did not contain another electrolyte and the sodium acetate buffer was used at $0.5 \mathrm{mM}$ because of the high conductivity of this solution. All the buffer solutions were adjusted to a $\mathrm{pH}$ of 3.9 by the stepwise addition of small amounts of acetic acid. The CE separation buffer was placed in the anode reservoir of the $C E$ instrument. The analytes dissolved in $50 \%(\mathrm{v} / \mathrm{v})$ methanol-water were injected from a separate reservoir by the pressure technique and the injection volume was about $50 \mathrm{~nL}$. The concentration of analytes was $50 \mathrm{ppm}$ and the amount injected was about $2.5 \mathrm{ng}$. The HV applied to the CE anode was generally $25 \mathrm{kV}$, but was lowered to $20 \mathrm{kV}$ with the more conductive sodium acetate buffer. The syringe pump was used to deliver $2 \mu \mathrm{L} / \mathrm{min}$ of $0.1 \%$ acetic acid in methanol to the $C E$ probe where it is used as a sheath liquid around the outside of the $C E$ capillary to provide a stable electrical connection between the tip of the $C E$ capillary and ground. The electrospray HV was -3000 
Table 1. Migration times and electro-osmotic $\left(\mu_{\mathrm{eg}}\right)$ and electrophoretic $\left(\mu_{\mathrm{e}_{p}}\right)$ mobilities as a function of the electrolyte in the CE separation buffer ${ }^{\mathrm{a}}$

\begin{tabular}{|c|c|c|c|c|c|c|}
\hline \multirow[b]{2}{*}{ CE electrolyte } & \multicolumn{2}{|c|}{ Phenol } & \multicolumn{2}{|c|}{ Paraquat } & \multicolumn{2}{|c|}{ Diquat } \\
\hline & $\underset{(\min )}{t}$ & $\begin{array}{l}\mu \quad \times 10^{4} \\
\left(\mathrm{~cm}^{2} / \mathrm{V} \mathrm{s}\right)\end{array}$ & $\begin{array}{c}t \\
(\mathrm{~min})\end{array}$ & $\begin{array}{c}\mu \quad \times 10^{4} \\
\left(\mathrm{~cm}^{2} / \mathrm{V} \mathrm{s}\right)\end{array}$ & $\stackrel{t}{t}$ & $\begin{array}{c}\mu \underset{10^{4}}{\left.\times \mathrm{cm}^{2} / \mathrm{V} \mathrm{s}\right)}\end{array}$ \\
\hline Acetic acid ${ }^{\mathrm{b}}$ & 22.13 & 1.63 & 6.42 & 3.99 & 6.42 & 3.99 \\
\hline Sodium acetate ${ }^{c}$ & 34.20 & 1.05 & 7.70 & 3.63 & 7.93 & 3.49 \\
\hline $\begin{array}{l}\text { Ammonium } \\
\text { bicarbonate }\end{array}$ & 32.53 & 1.11 & 7.61 & 3.63 & 7.86 & 3.48 \\
\hline Ammonium acetate & 36.79 & 0.98 & 7.75 & 3.68 & 7.96 & 3.55 \\
\hline Ammonium formate & 28.84 & 1.25 & 7.47 & 3.58 & 7.72 & 3.43 \\
\hline Ammonium citrate & 34.44 & 1.05 & 8.07 & 3.42 & 8.40 & 3.25 \\
\hline
\end{tabular}

Migration times were measured with a UV detector. CE separation buffers consisted of about $0.5-1.5 \%$ acetic acid plus the electrolyte at $5 \mathrm{mM}$ in $50 \%(\mathrm{v} / \mathrm{v})$ methanol-water adjusted to $\mathrm{pH} 3.9 \mathrm{with}$ acetic acid.

No additional electrolyte.

Concentration was $0.5 \mathrm{mM}$ in the separation buffer.

on the larger outer cylindrical electrospray electrode, -3400 on the smaller inner electrode, and -3800 on the Pt-coated end of the glass capillary that leads to the mass spectrometer. A flow of heated dry nitrogen gas (heater temperature $180^{\circ} \mathrm{C}$ ) was maintained counter to the electrospray flow. The spectrometer was repetitively scanned from $\mathrm{m} / \mathrm{z} 50-360$ at $0.3 \mathrm{~s} / \mathrm{scan}$ during the separation and detection. Before each change of buffer solution, the capillary was rinsed with $0.2-\mathrm{M}$ sodium hydroxide followed by reagent water and then by the new buffer solution.

\section{Direct Infusion Electrospray / Mass Spectrometry}

The equipment and conditions for direct infusion ES/MS were identical to those used for the CE-ES/MS except no HV was applied to the CE anode, the analytes were dissolved in separate solutions of the same series of buffers, and the analyte solutions were in- fused by using the pressure mode of the CE instrument.

\section{Capillary Electrophoresis Migration Time Measurements}

The UV detector was used for CE migration time measurements and no sheath liquid was employed. All separation buffers were identical to those used for CE-ES/MS. The total length of the uncoated fused silica capillary used for the migration time measurements was $82 \mathrm{~cm}$. The distance from the anode to the UV detector window was $66 \mathrm{~cm}$ and a positive $25 \mathrm{kV}$ was applied to the anode end of the capillary except for the sodium acetate buffer, which required $20 \mathrm{kV}$. The cathode end of the capillary was grounded. Electro-osmotic mobility was measured by the addition of $20 \mathrm{ppm}$ of phenol to the analytes in the same series of separation buffers and at the same $\mathrm{pH}$ as described in the foregoing text.

Table 2. Nominal relative abundances of the major ions in the CE-ES/MS of paraquat and diquat as a function of the electrolyte in the CE separation buffer ${ }^{2}$

\begin{tabular}{|c|c|c|c|c|c|c|}
\hline \multirow[b]{2}{*}{ CE electrolyte } & \multicolumn{3}{|c|}{ Paraquat ions $(m / z)$} & \multicolumn{3}{|c|}{ Diquat ions $(m / z)$} \\
\hline & 93 & 185 & $186^{b}$ & 92 & 183 & $184^{b}$ \\
\hline Acetic acid & 100 & 10 & 19 & 100 & 10 & 19 \\
\hline Sodium acetate & 100 & 10 & 8 & 100 & 50 & 3 \\
\hline $\begin{array}{l}\text { Ammonium } \\
\text { bicarbonate }\end{array}$ & 100 & 100 & 17 & 100 & 100 & 17 \\
\hline Ammonium acetate & 30 & 100 & 17 & 5 & 100 & 10 \\
\hline Ammonium formate & 20 & 100 & 7 & 20 & 100 & 7 \\
\hline Ammonium citrate & 5 & 100 & 7 & 5 & 100 & 7 \\
\hline
\end{tabular}




\section{Results and Discussion}

Analyte migration times $(t)$ were measured and electrophoretic mobilities $\left(\mu_{\mathrm{ep}}\right)$ and electro-osmotic mobilities $\left(\mu_{\mathrm{eo}}\right)$ were calculated to determine the effects of various separation buffers on these $C E$ parameters. Table 1 lists the measured and calculated values for phenol, paraquat, and diquat as a function of the CE separation buffer electrolytes. The migration times were measured with a UV detector because the migration time of a neutral marker compound is required to calculate the electro-osmotic mobility $\left(\mu_{\mathrm{eo}}\right)$ and neutral compounds typically cannot be detected with electrospray mass spectrometry. However exactly the same separation buffers were used for the migration time measurements and for the CE-ES/MS. Accordingly, $50 \%(\mathrm{v} / \mathrm{v})$ methanol-water that contained $0.5-1.5 \%$ acetic acid at $\mathrm{pH} 3.9$ was used in all the separation buffers because the electrospray ionization process is far more stable with this mixture than with the $100 \%$ aqueous buffers ordinarily used for CE migration time measurements. Phenol ( $\left.\mathrm{p} K_{a}=10\right)$ was selected as the neutral marker compound because it is readily available in pure form, easily detected in the UV, and is not ionized at $\mathrm{pH}$ 3.9.

The separation of paraquat and diquat is in the range of 12.5-20 s depending on the buffer composition, but no separation was observed with acetic acid alone (Table 1). The electrophoretic mobilities of paraquat and diquat are generally over three times larger than the electro-osmotic mobility as expected at a buffer $\mathrm{pH}$ of 3.9. The $\mu_{\mathrm{ep}}$ accounts for the relatively rapid and efficient separation of these two similar dications. Paraquat probably migrates slightly faster than diquat because paraquat's charge is more highly dispersed than diquat's, whose more localized charge attracts buffer anions somewhat more strongly which slightly reduces its electrophoretic mobility (Table 1). The best separation occurred with diammonium citrate, which provided the largest, the most highly charged, the highest charge density, and probably the tightest ion pairing buffer anion.

Figure 1 shows the separation and detection of paraquat and diquat by $\mathrm{CE}$-positive ion electrospray mass spectrometry in a buffer that contained ammonium acetate. The total ion electropherogram was acquired by repetitive scan of the range $m / z 50-360$, but Figure 1 is a display of the abundances of $m / z 183$ and 185 as a function of migration time. The migration times in this experiment are a little longer than those shown in Table 1 because of slightly different conditions, mainly a longer fused silica capillary was used with the electrospray interface.

Table 2 shows the nominal relative abundances of the major ions in the CE-ES positive ion mass spectra of paraquat and diquat as a function of the electrolyte in the CE separation buffer. All the separation buffers were the same except for the identity of the electrolyte and the sodium acetate concentration, which was one-

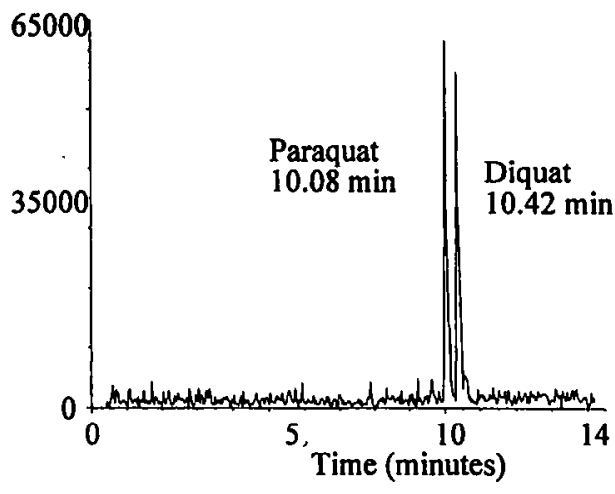

Figure 1. Extracted ion electropherogram for $m / z \quad 183$ and 185 from the capillary electrophoresis separation and positive ion electrospray mass spectrometry detection of paraquat and diquat.

tenth the concentration of the other electrolytes because of its high conductivity. All buffers were adjusted to $\mathrm{pH} 3.9$ with acetic acid. No other major ions were observed, but a few spectra showed low abundance (generally $<10 \%$ ) ions corresponding to solvent adducts or the loss of a methyl group from paraquat.

As seen in Table 2, the doubly charged molecular ions $\left(\mathrm{M}^{2+}\right)$ at $m / z 93$ and 92 dominate the CE-ES spectra with either acetic acid alone or with sodium acetate-acetic acid in the separation buffer. Singly charged molecular ions $\left(\mathrm{M}^{+}\right)$also are observed at $\mathrm{m} / \mathrm{z}$ 186 and 184, respectively, and these ions persist with modest abundances with the use of various buffer electrolytes (the relative abundances of the $\mathrm{m} / \mathrm{z} 186$ and 184 ions in Table 2 have been reduced to eliminate

${ }^{13} \mathrm{C}$ contributions from the $\mathrm{m} / z 185$ and 183 ions). The $\mathrm{M}^{+}$ions at $\mathrm{m} / z \quad 186$ and 184 must be formed by one-electron reductions of the $\mathrm{M}^{2+}$ ions. The reducing agent could be an anion, a component of the separation buffer, or electrons supplied in the CE or electrospray processes. Electron transfer processes, primarily oxidation, in electrospray have been observed and studied in recent years [5].

The singly charged ions at $m / z 185$ and 183 (Table 2) likely are formed by the deprotonation of the $\mathrm{M}^{2+}$ ions at $\mathrm{m} / z 93$ and 92 and are designated the $\left[\mathrm{M}^{2+}\right.$ $\left.\mathrm{H}^{+}\right]^{+}$ions. With just acetic acid in the methanol-water buffer, only very modest deprotonation occurs and the $\mathrm{M}^{2+}$ ions are the base peaks. With sodium acetateacetic acid as the separation buffer electrolyte, again very modest deprotonation of paraquat is observed, but deprotonation of diquat $(m / z 183)$ is enhanced. With ammonium bicarbonate as the separation buffer electrolyte, the $\mathrm{M}^{2+}$ ions and deprotonated ions are about the same abundance. With ammonium acetate, formate, and citrate the deprotonated ions become the base peaks and the relative abundances of the $\mathrm{M}^{2+}$ ions are reduced substantially with both compounds.

Direct infusion electrospray mass spectra of the analytes also were measured by using nearly identical conditions to ascertain whether the reduction and deprotonation reactions occurred primarily in the $C E$ or 
in the ES process. Direct infusion was accomplished by using the same instrumental arrangement by turning off the anode high voltage power supply, injecting the analytes separately but in the same series of buffer solutions, and by using the pressure mode of the CE instrument to infuse the sample into the electrospray source. With direct infusion, no ion abundances were observed at $\mathrm{m} / z 186$ and 184 that were greater than expected from ${ }^{13} \mathrm{C}$ contributions from the $m / z 185$ and 183 ions, respectively. Therefore we conclude that the one-electron reductions of the $\mathrm{M}^{2+}$ ions are inherent to the $C E$ and a consequence of the $C E$ current.

The base peaks in the direct infusion electrospray spectra followed the same general trend shown in Table 2, that is, the $\mathrm{M}^{2+}$ ions at $m / z 93$ and 92 were the base peaks in acetic acid alone and in the sodium acetate-acetic acid buffer. Also, in these buffers, the deprotonated ions at $\mathrm{m} / \mathrm{z} 185$ and 183 were only $11-22 \%$ relative abundance. With ammonium bicarbonate, ammonium acetate, and ammonium formate, the base peaks were the deprotonated ions at $\mathrm{m} / \mathrm{z} 185$ and 183 and the $\mathrm{M}^{2+}$ ions were $24-59 \%$ with only paraquat and ammonium bicarbonate giving as much as $77 \% \mathrm{~m} / \mathrm{z} 93$. These results indicate that the differences observed in relative abundances of the $\mathrm{M}^{2+}$ ions are deprotonated ions as a function of buffer are inherent to the electrospray process and not caused by reactions during the $\mathrm{CE}$ separation. The $\mathrm{M}^{2+}$ ions at $m / z 93$ and 92 have somewhat higher relative abundances in the direct infusion spectra with ammonium ion buffers than they have in the corresponding CE-ES spectra in Table 2. This may be accounted for by the fact that no $\mathrm{M}^{2+}$ ion-consuming one-electron reductions to the singly charged $m / z 186$ and 184 ions were observed in the direct infusion spectra.

Mirza and Chait [6] reported that in the electrospray of multiply charged peptides and proteins, deprotonation and reduction of the average charge state were influenced strongly by anionic species present in the electrospray solution. They also found that some anions (e.g., trifluoroacetate) were more effective deprotonating agents than others (e.g., chloride). They hypothesized that ion pairs-formed in solution-dissociate during the process of desolvation of charged droplets or in the gas phase to produce the neutral acid and the peptide with a reduced charge state. Their observations were made with direct infusion of sample into the ES source and without CE separation. Nevertheless, their observations suggest an analogous mechanism for the deprotonation of the paraquat and diquat $\mathrm{M}^{2+}$ ions.

Scheme I is suggested as the mechanism by which the relatively strong base ammonia removes a proton from the doubly charged molecular ion of paraquat (or diquat in an analogous process) during desolvation of charged electrospray droplets or in the gas phase. When ammonium ion is used in the $C E$ separation buffer, it must be present in relatively high concentrations in the electrospray because, like the positively

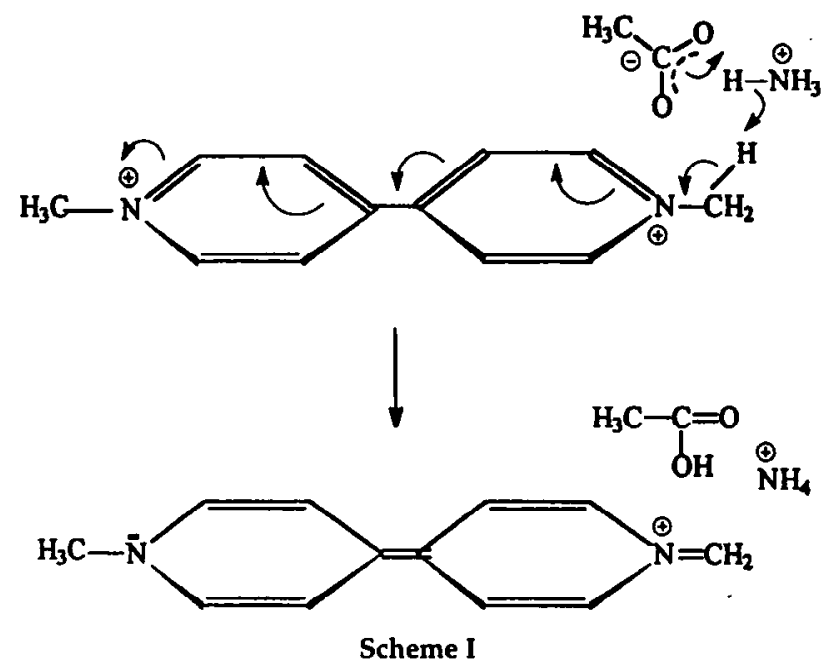

charged analytes, it migrates toward the $\mathrm{CE}$ cathode. Ammonium ion in aqueous solution is, of course, in an instantaneous equilibrium with ammonia and protonated water. Ammonia is therefore available to abstract an acidic proton from a methyl group of paraquat (or a methylene group of diquat) to give the deprotonated singly charged ion. Ammonia also could be formed by transfer of a proton from the ammonium ion to an anion, such as acetate, as illustrated in Scheme I. The extent to which buffer anions are present in the CE-ES charged droplets is unknown. Without a strong electro-osmotic flow, anions migrate toward the anode and at $\mathrm{pH} 3.9$ the electro-osmotic flow is weak (Table 1). Nevertheless some anions likely are present in the charged droplets and they also can serve as proton acceptors as in Scheme I. That buffer anions play some role in the deprotonation is indicated by the relative abundances observed with ammonium bicarbonate buffer (Table 2), where the degree of deprotonation is quite different than with the other ammonium ion buffer electrolytes. When acetic acid alone or sodium acetate-acetic acid are the electrolytes, all the available proton acceptors (for example, water or acetate) are apparently too weakly basic or too depleted to effectively deprotonate the doubly charged paraquat molecular ion. However acetate must be somewhat more effective with diquat which has more concentrated charge and probably somewhat more acidic protons (Table 2, sodium acetate buffer). Scheme I predicts that the proton is removed from one of the methyl groups or paraquat and from one of the methylene groups of diquat, a process that could be tested with suitably isotopically labeled analytes.

The CE-ES mass spectra of paraquat and diquat are relatively simple but they contain highly characteristic doubly and singly charged molecular ions and deprotonated ions. It is essential to understand and control the conditions and reactions that lead to the variable relative abundances of these ions if this technique is to become the basis for an environmental analytical method for these herbicides and related substances. 
Existing analytical techniques for these herbicides include chemical reduction to produce derivatives amenable to gas chromatography [7], immunoassay [8], separation by CE with UV absorbance detection [9], and square-wave voltammetry [10]. However all of the existing techniques are limited in their capabilities because they require an additional chemical derivatization step or special immunoassay reagents or use nonspecific detectors that are subject to false positive results.

The analytical potential of the CE-ES technique was investigated and found to be promising. The smallest quantity of paraquat or diquat measured in this work with a signal-to-noise ratio of at least 3 is $200 \mu \mathrm{g} / \mathrm{L}$. Application of a CE sample stacking or preconcentration technique is expected to reduce this detection limit by a factor of 10 or more. The precision of replicate injections averaged $8 \%$ relative standard deviation over the concentration range of $1-50 \mathrm{mg} / \mathrm{L}$. The linear calibration range is at least $1-50 \mathrm{mg} / \mathrm{L}$ with an $r^{2}=0.992$ or better. Further research is in progress and will be reported at a later date.

\section{Acknowledgments}

The authors wish to express their appreciation to David Kryak of the U.S. Environmental Protection Agency for assistance in preparing the electrospray interface for use. This research was supported in part by the appointment of Xinbei Song to a postdoctoral research fellowship administered by the Oak Ridge Institute for Science and Education through an interagency agreement between the U.S. Department of Energy and the U.S. Environmental Protection Agency.

\section{References}

1. Chemical and Engineering Netus, March 4, 1996, $\mathrm{p} 6$.

2. The NIST /EPA/NIH Mass Spectra Database, PC Version 4.0, U.S. Department of Commerce, National Institute of Standards and Technology, Standard Reference Data Program, Gaithersburg, MD 20899.

3. Smith, R. D.; Olivares, J. A.; Nguyen, N. T.; Udseth, H. R. Anal. Chem. 1988, 60, 436-441.

4. Fenn, J. B.; Mann, M.; Meng, C. K.; Wong, S. F.; Whitehouse, C. M. Science 1989, 246, 64-71.

5. Van Berkel, G. J.; Zhou, F. J. Am. Soc. Mass Spectrom. 1996, 7, 157-162 and references therein.

6. Mirza, U. A.; Chait, B. T. Anal. Chem. 1994, 66, 2898-2904.

7. Seiber, J. N.; Woodrow, J. E. Arch. Environ. Contam. Toxicol. 1981, 10, 133-149.

8. Van Emon, J.; Hammock, B.; Seiber, J. N. Anal. Chem. 1986, $58,1866-1873$.

9. Kaniansky, D.; Ivanyi, F.; Onuska, F. I. Anal. Chemt. 1994, 66, 1817-1824.

10. Zen, J.; Jeng, S.; Chen, H. Anal. Chem. 1996, 86, 498-502. 